\title{
Editorial
}

\section{New Food Processing Technologies and Food Safety}

\author{
Md. Latiful Bari, ${ }^{1}$ Alexandru Grumezescu, ${ }^{2}$ Dike O. Ukuku, ${ }^{3}$ \\ Gargi Dey, ${ }^{4}$ and Tatsuro Miyaji ${ }^{5}$ \\ ${ }^{1}$ University of Dhaka, Dhaka 1000, Bangladesh \\ ${ }^{2}$ Politehnica University of Bucharest, Bucharest, Romania \\ ${ }^{3}$ Eastern Regional Research Center, Wyndmoor, PA, USA \\ ${ }^{4}$ KIIT University, Bhubaneswar, India \\ ${ }^{5}$ Shizuoka Institute of Science and Technology, Shizuoka, Japan \\ Correspondence should be addressed to Md. Latiful Bari; latiful@du.ac.bd
}

Received 17 August 2017; Accepted 20 August 2017; Published 24 September 2017

Copyright ( 2017 Md. Latiful Bari et al. This is an open access article distributed under the Creative Commons Attribution License, which permits unrestricted use, distribution, and reproduction in any medium, provided the original work is properly cited.

The microflora of foods is very significant to food producers, processors, and consumers and the food manufacturers including distributors are responding to consumers' demand for food products that are safe, fresher, and convenient for use. In some cases foods may be improperly processed and/or contaminated with spoilage bacteria or human bacterial pathogens. Knowledge of the levels of bacteria in food systems before and after processing, as well as the impact of storage time and temperature on microbial populations of minimally processed foods, should provide guidance to the food industry, regulatory agencies, and consumers in implementing HACCP plans and good manufacturing practices (GMP). Consumers' demand for fresh, healthy nutritious foods has triggered food industries to look for alternative technologies that can produce higher quality food, ensure safety and reasonable cost for the consumer, and at the same time improve food safety by reducing or eliminating foodborne bacterial pathogens. Similarly, the alternative novel processing technologies might be used as tools to tailor foods with added or enhanced functional and nutritional values, to lower carbon footprint and substantially reduce water volumes used in heat-transfer processes. Some of these new processing concepts include advanced thermal and nonthermal technology that uses mechanical, electrical, and electromagnetic energy and hurdle (combined-applications) approaches. The different fundamental principles and the performance capabilities of some novel technologies and processes differ from traditional processing in terms of the types of food categories, microbial efficacy and destruction models, and desired and undesired effects on food quality and their economic and environmental impact were published in this issue. This special issue addresses 7 such new food processing technologies, including high pressure processing (HPP), electrical impedance spectroscopy (EIS), ultrasound, low water activity food, vacuum frying, innovative transduction process to supply safe fresh snack, and false labeling prevention technique.

High hydrostatic pressure processing (HPP) has been successfully applied to heat-sensitive drinks and solid foods such as jams and jellies, fruit juices, ham, cooked ready-toeat meat products, and seafood products such as oysters. In this special issue, H. Ogihara et al. showed the effectiveness of 400 and $500 \mathrm{MPa}$ of high hydrostatic pressure processing on raw beef liver which effectively kills pathogens and thus is safe for consumption, but this treatment also changes the texture of the raw beef liver. On the other hand, low HPP treated liver tissue became firmer, which changed to pale color that was considered unsuitable for raw consumption. In another study, O. A. Ijabadeniyi and Y. Pillay investigated the microbial safety of low water activity food and reported that certain low water activity foods may present a public health risk, despite fungal contamination. The spore-forming bacterium can be osmotolerant at both reduced and elevated temperatures. Thus they suggested that combination of other nonthermal treatments could be useful in controlling the safety and quality of low water activity foods. 
Electrical impedance spectroscopy (EIS) is an effective analytical technique to assess quality and safety of food and has been shown in wide application in food industries. In this special issue, X. Zhao et al. developed online EIS detection methods to replace traditional methods which save time, cost, and manpower and ensure quality grading of meat and fish. In another study, A. B. Oyedeji et al. described the impact of frying on texture and color of cassava root slices chips and suggested that vacuum frying at $118^{\circ} \mathrm{C}$ and $8 \mathrm{~min}$ could best preserve the quality attributes of cassava root slices chips. This simple but new technical piece of information could improve the product quality and consumers acceptance. In addition, L. M. Carrillo-Lopez et al. in their review article reported that the applications of ultrasound in food produce acoustic cavitations, which modifies the physical, chemical, and functional properties of food. The combination of ultrasound and sanitizing agent can improve the microbial reduction in foods and, thereby, retain their quality. However, they concluded that more research is still needed before applying this technology to a wider range of industrial sectors.

In another study, P.-Y. Chen described a dynamic mathematical model through which food adulteration or false labeling could be effectively prevented. He also discussed how the supply-demand factors in the food market are influenced by the administrative means that the sanitary inspectors have used to prevent false labeling of food. Furthermore, P.-Y. Chen constructed an innovative transduction process over the existing methods to assist snack food dealers or microfood enterprises in developing their food channels to supply safe fresh snack foods. This study also found that food safety assurance and providing sufficient nutrition information were the most essential topics that can influence consumers' decision-making regarding purchase of fresh snack foods.

Therefore, all the papers published in this special issue represent exciting, innovative, and applicable technologies in food safety and quality of various food, as well as emerging future research topics, in this multidisciplinary field. We hope that this special issue would attract major attention of the peers.

\section{Acknowledgments}

We would like to express our appreciation to all the authors, reviewers, and editors for their great support and contribution to making this special issue possible.

Md. Latiful Bari

Alexandru Grumezescu

Dike O. Ukuku

Gargi Dey

Tatsuro Miyaji 

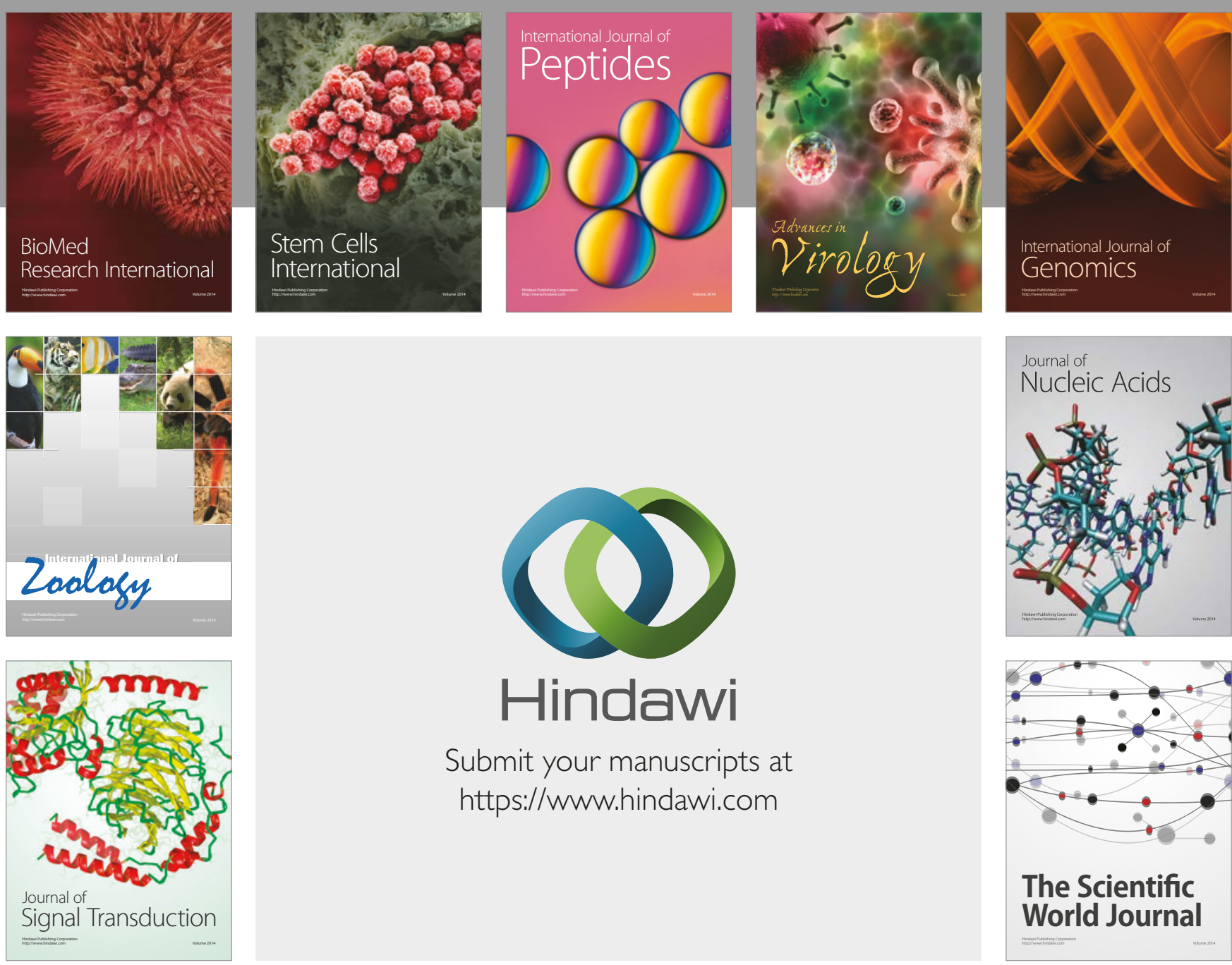

Submit your manuscripts at

https://www.hindawi.com
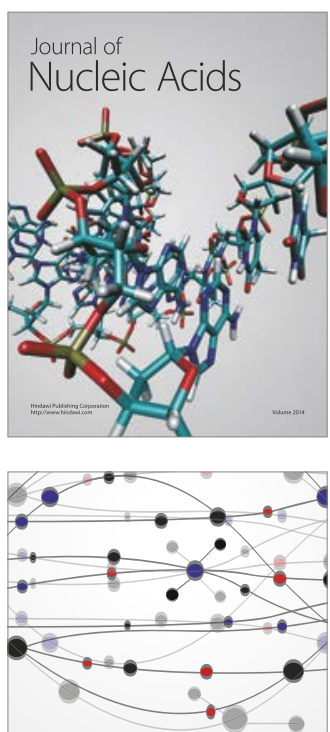

The Scientific World Journal

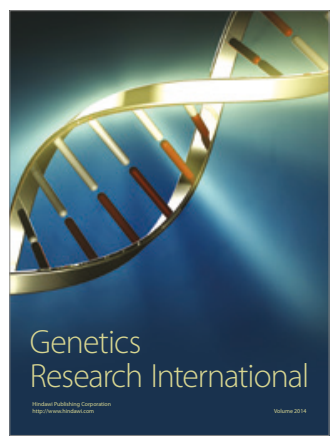

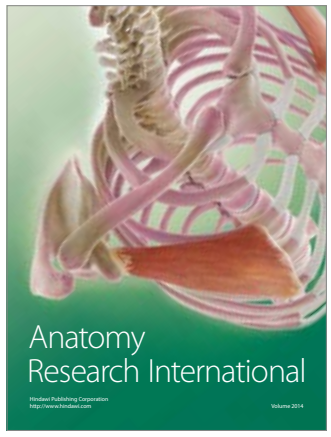

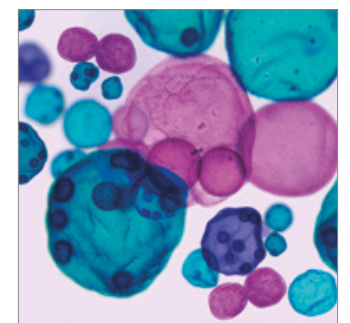

International Journal of Microbiology
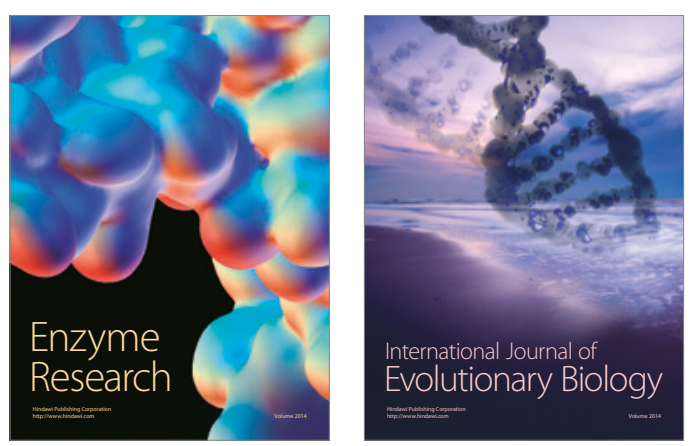
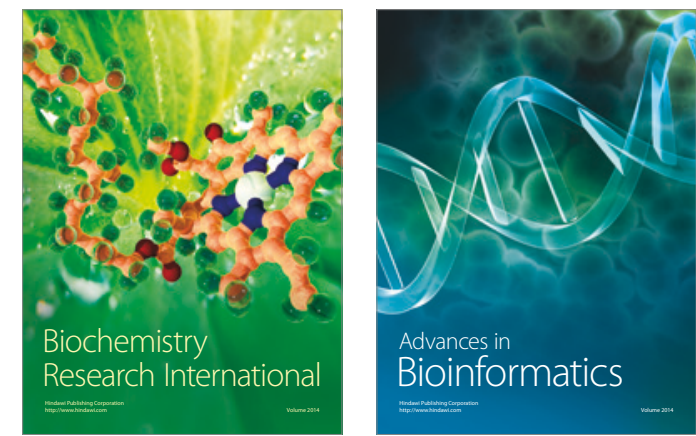

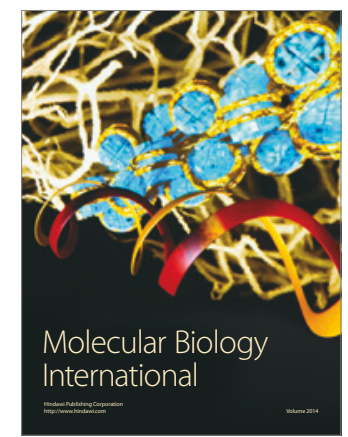

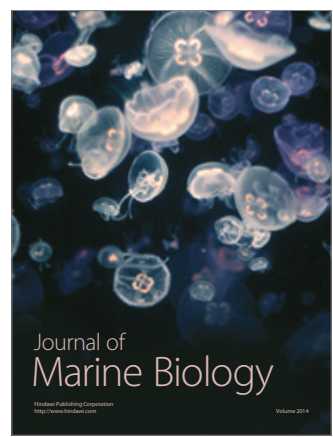

